\title{
Applying the WHO-ICRC BEC course to train emergency and inpatient healthcare workers in Sierra Leone early in the COVID-19 outbreak
}

Paul D Sonenthal ${ }^{1,2,3^{*}}$ (D) Chiyembekezo Kachimanga ${ }^{4}$, Doris Komba ${ }^{5}$, Moses Bangura ${ }^{4}$, Nicholas Ludmer ${ }^{6}$, Marta Lado ${ }^{4}$, Marta Patino ${ }^{4}$, Rachel B Gerrard ${ }^{3}$, Matthew J Vandy ${ }^{5}$, Regan H Marsh ${ }^{2,3,7}$, Joia Mukherjee ${ }^{2,3}$ and Shada A Rouhani ${ }^{2,3,7}$

\begin{abstract}
Background: Treating critical illness in resource-limited settings during disease outbreaks is feasible and can save lives. Lack of trained healthcare workers is a major barrier to COVID-19 response. There is an urgent need to train healthcare workers to manage COVID-19. The World Health Organization and International Committee of the Red Cross's Basic Emergency Care course could provide a framework to cross-train personnel for COVID-19 care while strengthening essential health services.

Methods: We conducted a prospective cohort study evaluating the Basic Emergency Care course for healthcare workers from emergency and inpatient units at two hospitals in Sierra Leone, a low-income country in West Africa. Baseline, post-course, and six month assessments of knowledge and confidence were completed. Questions on COVID-19 were added at six months. We compared change from baseline in knowledge scores and proportions of participants "very comfortable" with course skills using paired Student's t-tests and McNemar's exact tests, respectively.

Results: We enrolled 32 participants of whom 31 completed pre- and post-course assessments. Six month knowledge and confidence assessments were completed by 15 and 20 participants, respectively. Mean knowledge score post-course was $85 \%$ (95\% Cl: $82 \%$ to $88 \%$ ), which was increased from baseline $(53 \%, 48 \%$ to $57 \%$, $p$-value $<0.001)$. There was sustained improvement from baseline at six months (73\%, 67\% to $80 \%$, $p$-value 0.001$)$. The percentage of participants who were "very comfortable" performing skills increased from baseline for 27 of 34 skills post-training and 13 skills at six months. Half of respondents strongly agreed the course improved ability to manage COVID-19.

Conclusions: This study demonstrates the feasibility of the Basic Emergency Care course to train emergency and inpatient healthcare workers with lasting impact. The timing of the study, at the beginning of the COVID-19 pandemic, provided an opportunity to illustrate the strategic overlap between building human resource capacity for long-term health systems strengthening and COVID-19. Future efforts should focus on integration with national training curricula and training of the trainers for broader dissemination and implementation at scale.
\end{abstract}

Keywords: Emergency and critical care, COVID-19, Basic emergency care course, Capacity building, Training

*Correspondence: p.sonenthal@gmail.com

${ }^{1}$ Brigham and Womens Hospital, Division of Pulmonary and Critical Care

Medicine, 75 Francis Street Massachusetts, Boston, MA 02115, USA

Full list of author information is available at the end of the article

\section{Introduction}

The West African Ebola virus disease epidemic of 20142016 highlighted the vulnerability of the region's chronically understaffed and underresourced health systems $[1$, 2]. One of the major barriers to controlling the epidemic 
in Sierra Leone, Guinea, and Liberia was lack of trained healthcare workers [2]. During the epidemic, the Sierra Leone Ministry of Health and Sanitation (MoHS) developed a long-term strategy for strengthening the national health system which included healthcare worker career development and capacity building as one of four pillars [1]. Today, global shortages of human resources for health present an ongoing challenge as hospitals around the world struggle to maintain essential services while also providing care for patients with severe and critical COVID-19.

Data from the Ebola virus disease epidemic demonstrated that providing critical care in resource-limited settings during disease outbreaks is feasible and can save lives [3]. With approximately $18.5 \%$ of COVID-19 cases progressing to severe or critical disease [4], the provision of emergency and critical care services is essential. Unfortunately, there is a significant outcome gap in Africa relative to the rest of the world, with excess mortality following intensive care unit admission for COVID19 estimated between 11 and 23 deaths for every 100 patients [5]. Effective COVID-19 care depends in part on the ability to recognize severe illness and rapidly deliver life-saving interventions. The scarcity of skilled healthcare workers in many low-income countries (LICs), where emergency and critical care training is limited, poses a significant challenge for COVID-19 response, and is likely one of several factors contributing to outcome disparities.

Meeting the urgent need to identify methods for training multiple cadres of healthcare workers to manage COVID-19 patients has potential benefits that extend well beyond the pandemic. Training healthcare workers to recognize, assess, and stabilize emergency and critical conditions will not only help fight current and future epidemics but will also strengthen the long-term delivery of comprehensive healthcare in low- and-middle-income countries [6] where $54 \%$ of annual deaths are from conditions potentially treatable by prehospital and facilitybased emergency care [7].

The World Health Organization (WHO) and International Committee of the Red Cross's Basic Emergency Care Course (BEC) is an open-access five-day course for front-line emergency care providers in resource-limited settings [8] that has been implemented in multiple countries including Ethiopia [9], Zambia [10], Uganda [11], Tanzania [11], and Nigeria [12]. The BEC employs lectures and hands-on skill-based exercises to train providers in the assessment and management of emergency conditions [8]. Typically, BEC courses only enrol emergency unit staff, but the concepts and skills are relevant to inpatient management as well. The BEC course's concepts could serve as a foundation both to cross-train personnel for COVID-19 care and to help maintain and strengthen essential health services in face of the pandemic. In early 2020 , just prior to the start of the global pandemic we taught an expanded version of the BEC course in Sierra Leone, a LIC in West Africa, targeting healthcare workers from medical inpatient wards and intensive care units in addition to emergency units. To evaluate the impact of the course on healthcare workers' knowledge and confidence in providing emergency and critical care services, we conducted a prospective cohort study of course participants, which coincided with the onset of the COVID19 pandemic.

\section{Methods \\ Setting}

We conducted a prospective cohort study at two hospitals in Sierra Leone, a West African country of over 7.5 million people [13]. Ranked $182^{\text {nd }}$ of 189 countries by the Human Development Index [14] and with a life expectancy that is only 54.7 years [14], Sierra Leone is among the least developed countries worldwide. The 2014-2016 Ebola virus disease epidemic devastated an already fragile health system-staff losses and shaken community confidence in health facilities led to further deterioration of health services and outcomes [15]. The trainings were held at two hospitals in Sierra Leone-Koidu Government Hospital (KGH) and Connaught Hospital. KGH is a public secondary hospital with 170 beds located in Kono, a rural district in eastern Sierra Leone, with a catchment population of 506,100 as of the most recent 2015 census [13]. Connaught Hospital, located in the capital of Freetown, has 300 beds and serves as the national's main public tertiary hospital. The course content and study design were finalized prior to December 2019, when the first reports of COVID-19 became public.

The Sierra Leone MoHS has emphasized that lack of human resources is "a major contributor to the poor health outcomes seen in Sierra Leone," and identified "ongoing in-service training and support for all cadres" of healthcare workers as a key priority [16]. A nationwide shortage of doctors in Sierra Leone is exacerbated by their disproportionate concentration in urban areas. For example, Freetown has 1.1 doctors per 10,000 population [17] while Kono District has 0.08 doctors per 10,000 population [17]. In contrast, Community Health Officersadvanced practice providers with 3 years of training - are more evenly distributed across the country and are the principal frontline clinicians in many rural areas [17].

Emergency and critical care services are underdeveloped in Sierra Leone. An assessment of seven hospitals in Freetown found significant deficiencies in emergency and critical care services across multiple domains [18]. Only $67 \%$ of facilities had provided training to staff in adult 
emergency care and 50\% had provided training for adult triage [18]. Sierra Leone does not have a formal postgraduate training program in emergency or critical care [18].

\section{Course structure}

The BEC course is a five day, in-person training consisting of lectures and hands-on skills stations [8]. The course emphasizes a systematic method for assessment and stabilization of all patients using the ABCDE (airway, breathing, circulation, disability, and exposure) approach [8]. The first day is an introduction to the ABCDE approach with each of the subsequent four days focussed on applying this approach to specific conditions (Fig. 1). Although developed prior to the COVID-19 pandemic, much of the BEC course content has direct relevance to COVID-19. Notably, the third day is devoted to acute presentations of difficulty in breathing. Specific sessions include lectures on assessment, evaluation, and management; interactive case presentations; and hands-on teaching of essential skills such as basic airway manoeuvres, basic airway device insertion, oxygen administration, and bag-valve-mask ventilation. The training courses in Kono and Freetown were conducted sequentially during February 2020 in collaboration with the non-profit organization Partners In Health (PIH). Attendance and successful completion of all course components, demonstrated proficiency in all skill stations, and a score above $75 \%$ on the post-course knowledge assessment were required to pass the course. Four facilitators delivered the courses, with a trainer to trainee ratio of 1:3.8 and 1:4.5 for Kono and Freetown, respectively. The facilitators included two physicians from the United States and one clinician and one nurse from Sierra Leone. Facilitators were selected based on leadership ability, prior teaching experience, and emergency and critical care experience. Both facilitators from Sierra Leone had previously taken the BEC course. Content covered over the five days of the courses included all core BEC content.

\section{Participants}

Nurses, community health officers, and doctors providing clinical care for critically ill patients in emergency or inpatient settings in Sierra Leone were eligible for invitation to the training. Participants were nominated by staff from PIH and MoHS. Eligibility was not restricted to Connaught Hospital or $\mathrm{KGH}$ - participants could come from any public sector hospital in Sierra Leone. All participants were over the age of 18 . The Partners Institutional Review Board (Protocol\#: 2020P000209) and Sierra Leone's National Ethics and Scientific Review Committee provided ethical approval.

\section{Data collection}

Pre- and immediate post-course assessments of knowledge and confidence are an essential component of the BEC course and were administered to all participants regardless of their participation in the study. On the first day of each training, all participants were approached for written informed consent and only data from those who provided consent were included in the study. Pretraining assessments were administered on the first day of each course and immediate post-training assessments were administered on the final day of each course. Both pre- and immediate post-training assessments were completed using paper forms. To assess knowledge retention and durability of impact, study participants were also asked to complete knowledge and confidence assessments at six months. Recognizing that the COVID-19 pandemic had created an increased demand for emergency and critical care services between initial study design and the pre-planned six month assessment, we developed a questionnaire with ten items related to COVID-19 and amended the study protocol to administer the COVID-19 questionnaire, in addition to the preplanned knowledge and confidence assessments, at six months.

Data were managed using REDCap electronic data capture tools [19]. Results from completed paper versions of the pre- and immediate post-course knowledge and confidence assessments were manually entered into the REDCap database. The six month follow-up was conducted by sending participants links to online versions of the knowledge, confidence, and COVID-19 assessments via email and SMS/WhatsApp messages using the survey function in REDCap. Two weeks later, participants yet to complete the six month assessments were sent reminders via phone call and SMS/WhatsApp.

\section{Instruments}

We assessed participant knowledge pre-course, immediately post-course, and at six months using a multiplechoice assessment containing 25 questions on course content which is a standardized component of the BEC course materials. We reported participant data as the percent of questions answered correctly.

Participant confidence was also assessed pre-course, immediately post-course, and at six months. Using a standardized instrument, participants rated their confidence performing 34 course skills divided into the domains of 1) overall confidence and skills and 2) specific skills. For each skill, participants rated their confidence on a Likert-scale of 1 ("not comfortable") to 7 ("very comfortable"). For each skill, we reported the number and proportion of participants responding "very comfortable". 


\begin{tabular}{|c|c|}
\hline Time & Session \\
\hline \multicolumn{2}{|c|}{ DAY 1: ABCDE AND SAMPLE } \\
\hline 8:30-8:45 & Introduction (welcome, brief outline, and logistics of course) \\
\hline 8:45-10:00 & Pre-course assessment (knowledge and confidence) \\
\hline 10:00-10:30 & Introduction: Why emergency care? (approach to patient, non-linear thinking, chief complaint based) \\
\hline 10:30-10:45 & Break \\
\hline 10:45-11:30 & ABCDE \\
\hline 11:30-12:15 & ABCDE: Pediatric considerations / SAMPLE \\
\hline 12:15-13:15 & Lunch \\
\hline 13:15-13:45 & Introduction to case-based discussion and learning with case / icebreaker \\
\hline 13:45-14:45 & ABCDE small group exercise / case scenarios \\
\hline 14:45-15:00 & Break \\
\hline 15:00-16:30 & Airway skills \\
\hline $16: 30-17: 00$ & Wrap-up/summary \\
\hline \multicolumn{2}{|c|}{ DAY 2: TRAUMA } \\
\hline 8:30-8:45 & Welcome/recap \\
\hline 8:45-9:30 & Trauma part I \\
\hline 9:30-9:45 & Break \\
\hline 9:45-10:30 & Trauma part II \\
\hline 10:30-11:00 & Small group excercises / case scenarios \\
\hline 11:00-12:00 & Skills: Extended physical examination \\
\hline 12:00-13:00 & Lunch \\
\hline 13:00-13:30 & Handover/transfer \\
\hline 13:30-14:45 & Immobilization skills: C-spine, log roll, full spine immobilization, immobilization of pregnant patient \\
\hline 14:45-15:00 & Break \\
\hline 15:00-16:30 & Wound management: General, snake bite, pelvic binder, recovery position \\
\hline $16: 30-17: 00$ & Wrap-up/summary \\
\hline \multicolumn{2}{|c|}{ DAY 3: DIFFICULTY IN BREATHING } \\
\hline $8: 30-8: 45$ & Welcome / recap \\
\hline 8:45-9:30 & Difficulty breathing: ABCDE, SAMPLE, causes \\
\hline 9:30-9:45 & Break \\
\hline 9:45-10:30 & Difficulty breathing: Management, pediatric considerations \\
\hline 10:30-11:00 & Difficulty breathing group excercises / case scenarios \\
\hline 11:00-12:00 & Breathing skills: breathing exam, supplemental 02 \\
\hline 12:00-13:00 & Lunch \\
\hline 13:00-15:00 & Breathing Skills: Needle decompression, spacers, BVM, three-sided dressings \\
\hline 15:00-15:30 & Break \\
\hline 15:30-16:30 & Simulation (one trauma case, one medical case) \\
\hline $16: 30-17: 00$ & Wrap-up/summary \\
\hline \multicolumn{2}{|c|}{ DAY 4: SHOCK } \\
\hline 8:30-8:45 & Welcome/recap \\
\hline 8:45-9:30 & Shock part I \\
\hline 9:30-9:945 & Break \\
\hline 9:45-10:30 & Shock part II \\
\hline 10:30-11:00 & Shock group exercise / case scenarios \\
\hline 11:00-12:00 & Burn management / fluid administration \\
\hline 12:00-13:00 & Lunch \\
\hline 13:00-15:00 & Circulation skills: Circulation exam, external bleeding control, uterine massage, IVs \\
\hline 15:00-15:30 & Break \\
\hline 15:30-16:30 & Simulation: Sepsis and burns \\
\hline 16:30-17:00 & Wrap-up / summary \\
\hline \multicolumn{2}{|c|}{ DAY 5: ALTERED MENTAL STATUS } \\
\hline $8: 30-8: 45$ & Welcome/ recap \\
\hline 8:45-9:30 & Altered mental status: History and exam \\
\hline 9:30-9:45 & Break \\
\hline 9:45-10:30 & Altered mental status: Causes and management \\
\hline 10:30-11:00 & AMS group excercise / case scenarios \\
\hline $11: 00-12: 00$ & Medication administration game \\
\hline $12: 00-13: 00$ & Lunch \\
\hline 13:00- 15:00 & Post-course assessment (knowledge and confidence) \\
\hline $15: 00-15: 15$ & Break \\
\hline 15:15-16:15 & Wrap-up / summary / feedback / certificates \\
\hline
\end{tabular}

Fig. 1 Course content and schedule 
This outcome was selected for consistency with prior studies $[10,12,20]$. For the COVID-19 assessment, a group of content experts developed and refined ten questions on experience and comfort treating COVID-19. These questions were completed as part of the six month assessment. Four were "yes/no" questions, two asked participants to rate their agreement with statements on a Likert scale of 1 ("strongly disagree") to 7 ("strongly agree"), and the remaining four asked participants to rate specific COVID-19 skills on a Likert-scale of 1 ("not comfortable") to 7 ("very comfortable"). We report the number and proportion of participants responding "yes", "strongly agree", or "very comfortable".

\section{Statistical analysis}

Data were analysed in Stata (Release 16). All participants that completed the course (i.e., attended all five days and completed immediate post-course knowledge and confidence assessments) were included in analyses. A passing score on the post-course knowledge assessment was defined as greater than $75 \%$ (at least 19 out of 25 questions) correct. Categorical variables were described using frequencies and proportions. Given the differences in training sites (urban tertiary vs. rural secondary) we also broke down outcome reporting by training site. Continuous variables were summarized by means, $95 \%$ confidence intervals, medians, and interquartile ranges. We compared mean test scores using paired Student's t-tests and proportions of participants "very comfortable" with course skills using McNemar's exact chi-squared tests for paired data. We used Wilcoxon matched-pairs signedrank test for comparing the median number of skills participants rated "very comfortable".

In additional analyses we compared mean knowledge scores by follow-up status using a two sided Student's $\mathrm{t}$-test. We also compared median number of skills participants rated "very comfortable" by follow-up status using Wilcoxon rank-sum test.

\section{Results}

\section{Participant characteristics}

We enrolled all 32 eligible participants, 14 (44\%) in Kono training and 18 (56\%) in Freetown, all of whom attended the first day of the course and completed baseline assessments. All participants approached for consent agreed to participate in the study. An additional course participant joined after the pre-course assessments and was not eligible for enrolment. A total of 31 (97\%) participants completed all five days of the course and immediate postcourse knowledge and confidence assessments and were included in analyses (Fig. 2).

Most participants were nurses (84\%), followed by community health officers (10\%), and doctors (3\%).
Participants from the Kono training worked in the medical ward (54\%), emergency unit (38\%), and intensive care unit (8\%). Participants from the Freetown training worked in the medical ward (61\%), emergency unit (22\%), and intensive care unit (17\%). At six months, followup confidence assessment data-including additional COVID-19 questions-were collected from 20 (65\%) participants and follow-up knowledge assessment data were collected from 15 (48\%) participants (Table 1). The median time from completion of the course to follow-up was 187 days (IQR: 172 to 234 ).

\section{Knowledge assessment}

Immediately post-training, there was a significant increase in knowledge assessment score among the Kono and Freetown groups compared to baseline (Table 2, Fig. 3) with all participants scoring above the required $75 \%$ to pass the course. The Kono group improved from a baseline mean score of 55\% (95\% CI: $48 \%$ to $61 \%$ ) to $83 \%$ ( $78 \%$ to $89 \%, \mathrm{p}<0.001)$ immediate post-training. The Freetown cohort improved from a mean score of $51 \%$ ( $44 \%$ to $58 \%)$ at baseline to $86 \%(83 \%$ to $90 \%, p<0.001)$ immediate post-training. Overall, participant scores increased by a mean of $33 \%$ ( $28 \%$ to $38 \%$ ) from baseline to immediate post-training. Among the 15 participants who completed the six month follow-up knowledge assessment, there was a sustained mean improvement of $19 \%$ (11\% to $28 \%$, p-value 0.001 ) from baseline (Fig. 4). However, compared to the immediate post-course assessment there was a mean decrease of $15 \%$ (9\% to $21 \%$, $p$-value $<0.001)$. Compared to participants who were lost to follow-up, participants who completed six month follow-up had similar baseline knowledge assessment scores but higher post-course scores (Table S1).

\section{Confidence assessment}

At baseline, individual participants reported feeling "very comfortable" performing a median of 9 (1 to 13) of the 34 skills covered in the course. Immediately post-training, the median number of "very comfortable" skills for individual participants increased to 25 ( 11 to $32, p<0.001$ ). This increase from baseline persisted at six months, with participants reporting a median of 21 (16 to 24) "very comfortable" skills ( $p$-value $<0.001)$. The overall proportion of participants who felt "very comfortable" increased for 27 of the course skills immediately post-training (Tables 3 and S2). Significant improvements were seen in skills relevant to COVID-19 including assessment and management of patients with difficulty breathing, fever, chest pain, and altered mental status as well as skills related to management of airway and breathing pathology (Table 3). Initial increases in proportion of "very comfortable" participants were sustained at six month follow-up for 12 (44\%) skills. In 


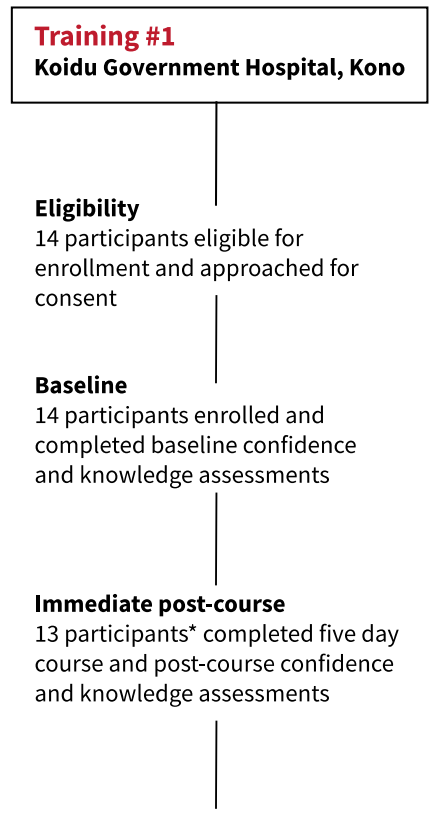

Six month follow-up

6 participants completed six month confidence, COVID-19, and knowledge assessments

1 participant completed six month confidence and COVID-19 assessments only

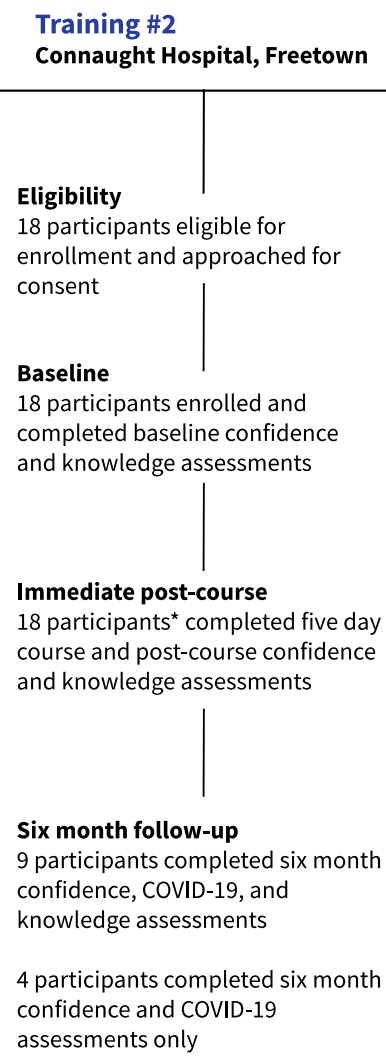

Immediate post-course

18 participants ${ }^{*}$ completed five day course and post-course confidence and knowledge assessments

\section{Six month follow-up}

9 participants completed six month confidence, COVID-19, and knowledge assessments

4 participants completed six month confidence and COVID-19 assessments only

Fig. 2 Participant enrolment

*Included in primary analyses

Table 1 Participant characteristics

\begin{tabular}{|c|c|c|c|}
\hline & $\begin{array}{l}\text { Kono } \\
(n=13)\end{array}$ & $\begin{array}{l}\text { Freetown } \\
(n=18)\end{array}$ & $\begin{array}{l}\text { Combined } \\
(n=31)\end{array}$ \\
\hline Completed immediate post-training confidence assessment n (\%) & $13(100 \%)$ & $18(100 \%)$ & $31(100 \%)$ \\
\hline Completed immediate post-training knowledge assessment n (\%) & $13(100 \%)$ & $18(100 \%)$ & $31(100 \%)$ \\
\hline Completed month 6 confidence assessment n (\%) & $7(54 \%)$ & $13(72 \%)$ & $20(65 \%)$ \\
\hline Completed month 6 knowledge assessment n (\%) & $6(46 \%)$ & $9(50 \%)$ & $15(48 \%)$ \\
\hline \multicolumn{4}{|l|}{ Work area } \\
\hline Intensive care unit n (\%) & $1(8 \%)$ & $3(17 \%)$ & $4(13 \%)$ \\
\hline Emergency unit n (\%) & $5(38 \%)$ & $4(22 \%)$ & $5(16 \%)$ \\
\hline Medical ward n (\%) & $7(54 \%)$ & $11(61 \%)$ & $22(71 \%)$ \\
\hline \multicolumn{4}{|l|}{ Role } \\
\hline Nurse n (\%) & $10(77 \%)$ & $16(89 \%)$ & $26(84 \%)$ \\
\hline Community health officer n (\%) & $3(23 \%)$ & 0 & $3(10 \%)$ \\
\hline Doctor n (\%) & 0 & $1(6 \%)$ & $1(3 \%)$ \\
\hline Other n (\%) & 0 & $1(6 \%)$ & $1(3 \%)$ \\
\hline
\end{tabular}

secondary analyses comparing proportion of participants with comfort ratings $\geq 6$ (out of 7), increases were sustained at six month follow-up for 10 skills (Table S3). The percentage of participants feeling "very comfortable" with oxygen administration at baseline (61\%) was significantly increased at six month follow-up (95\%, p-value 0.03). 
Table 2 Knowledge assessment scores

\begin{tabular}{|c|c|c|c|c|c|c|c|c|}
\hline & \multicolumn{2}{|c|}{ Baseline } & \multicolumn{3}{|c|}{ Post-training } & \multicolumn{3}{|c|}{ Six months } \\
\hline & $n$ & mean $(95 \% \mathrm{Cl})$ & $n$ & mean $(95 \% \mathrm{Cl})$ & $p$-value* & $n$ & mean $(95 \% \mathrm{Cl})$ & $p$-value^ ${ }^{\wedge}$ \\
\hline Kono & 13 & $55 \%$ (48\% to $61 \%)$ & 13 & $83 \%$ (78\% to 89\%) & $<0.001$ & 6 & $77 \%$ (62\% to $92 \%)$ & 0.07 \\
\hline Freetown & 18 & $51 \%$ (44\% to $58 \%$ ) & 18 & $86 \%$ (83\% to $90 \%)$ & $<0.001$ & 9 & $71 \%$ (63\% to $78 \%)$ & 0.008 \\
\hline Combined & 31 & $53 \%(48 \%$ to $57 \%)$ & 31 & $85 \%$ (82\% to $88 \%)$ & $<0.001$ & 15 & $73 \%$ (67\% to $80 \%)$ & 0.001 \\
\hline
\end{tabular}

${ }^{*}$ Two-sided paired t-test compared to baseline

$\wedge$ Two-sided paired t-test compared to baseline

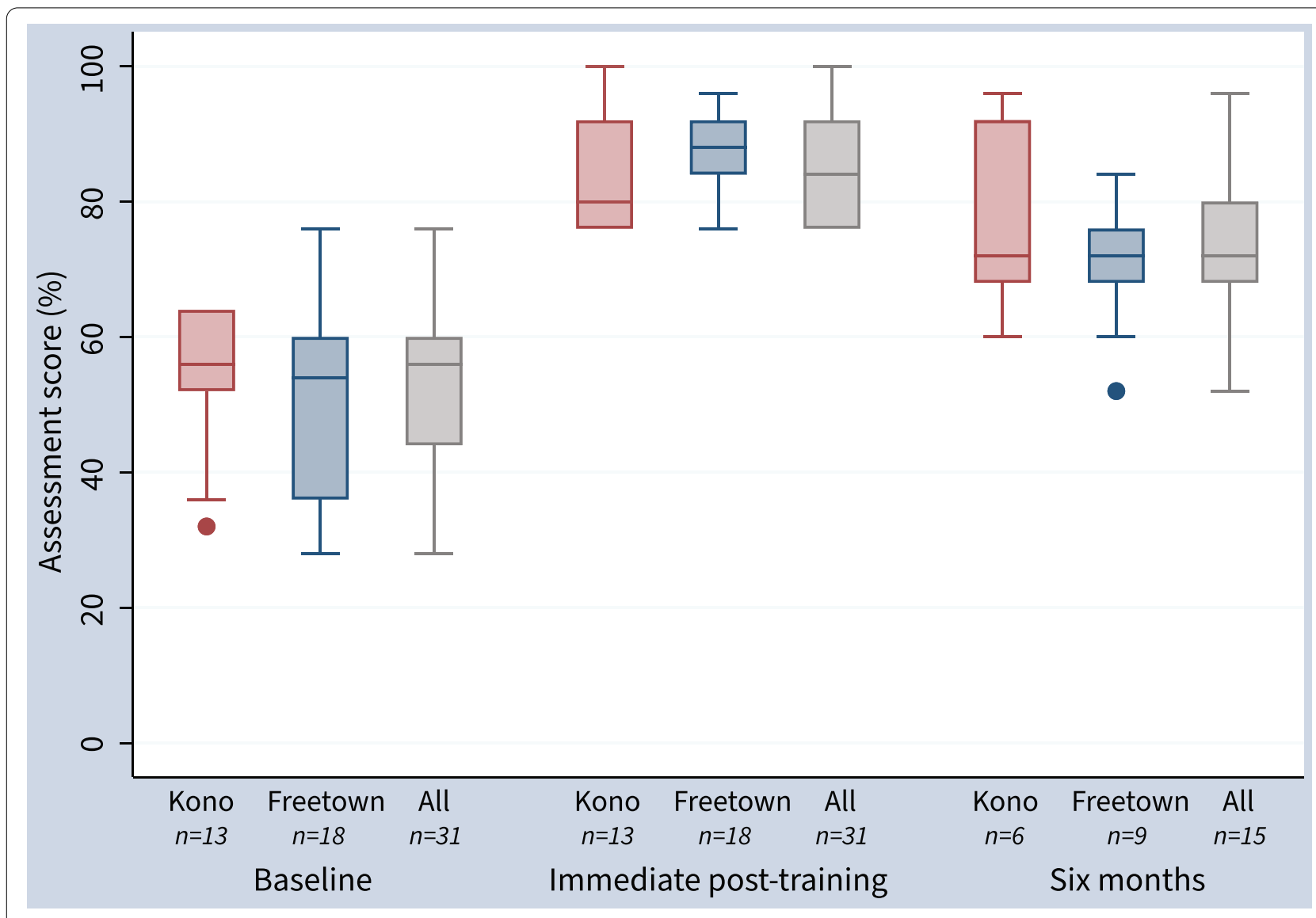

Fig. 3 Knowledge assessment score over time by training group, Box plots of assessment scores by group and follow-up

\section{COVID-19 assessment}

At six months, 8 (40\%) participants reported caring for patients with COVID-19 and 18 (90\%) reported caring for patients with suspected COVID-19. Half the participants who completed the six month followup assessment and $63 \%$ of those who had cared for patients with confirmed COVID-19 strongly agreed that the course improved their ability to manage patients with COVID-19 (Table 4). Further, $60 \%$ of participants reported they were "very comfortable" recognizing signs of severe COVID-19 and 55\% were "very comfortable" assessing respiratory status of patients with confirmed or suspected COVID-19. Among participants who had cared for patients with confirmed COVID-19, 75\% were very comfortable with both of these skills.

\section{Discussion}

This prospective cohort study assessing the impact of the BEC course at two hospitals in Sierra Leone early in the COVID-19 outbreak found improvements from baseline in participant knowledge and confidence in emergency 


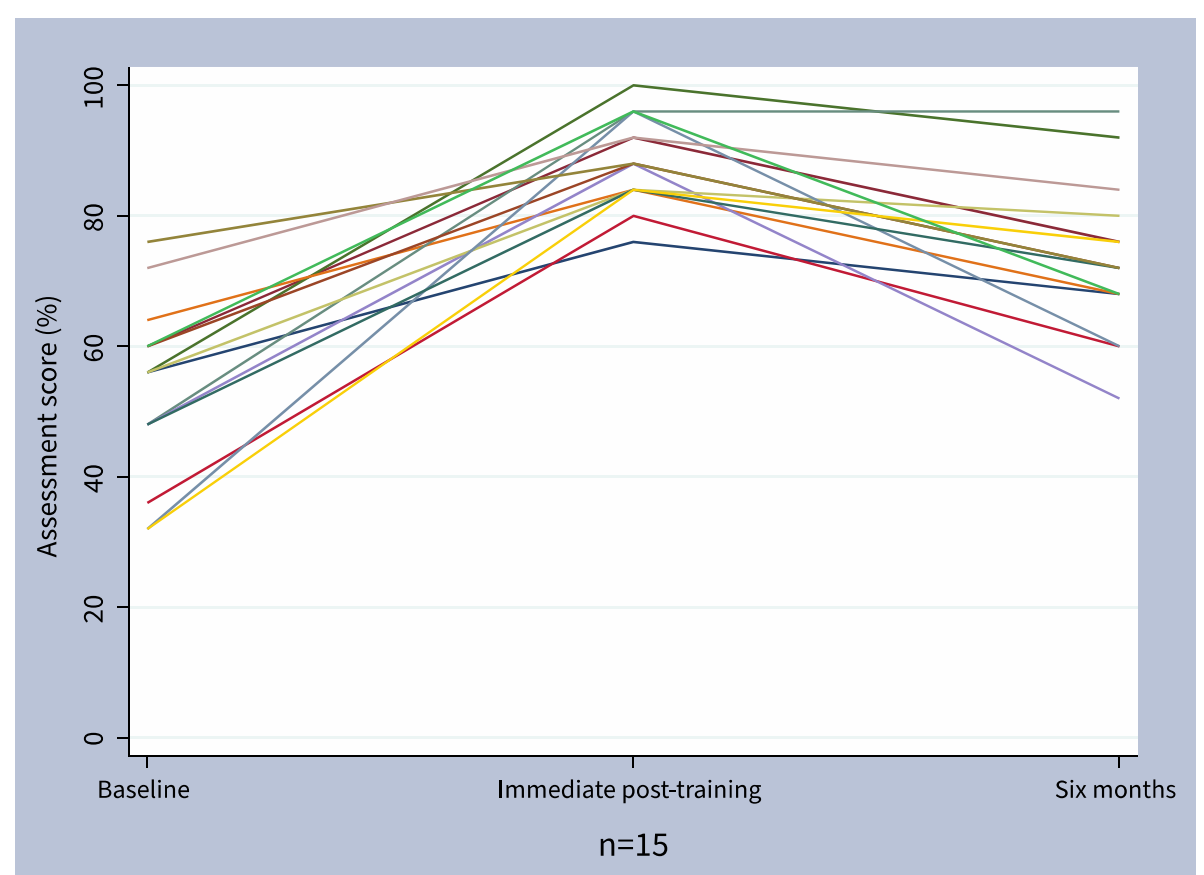

Fig. 4 Individual knowledge assessment scores over time, Each line represents one of the 15 participants with complete data for baseline, immediate-post training, and six month knowledge assessments

Table 3 Confidence assessment ratings of "very comfortable" for selected skills

\begin{tabular}{|c|c|c|c|c|c|}
\hline & \multirow{2}{*}{$\begin{array}{l}\text { Baseline } \\
(n=31) \\
n(\%)\end{array}$} & \multicolumn{2}{|c|}{$\begin{array}{l}\text { Post-training } \\
(n=31)\end{array}$} & \multicolumn{2}{|c|}{$\begin{array}{l}\text { Six months } \\
(n=20)\end{array}$} \\
\hline & & $n(\%)$ & $p$-value* & $n(\%)$ & $p$-value^ \\
\hline Assessing patients in an emergency department & $11(35 \%)$ & $19(61 \%)$ & 0.02 & $14(70 \%)$ & 0.11 \\
\hline Determining if a patient is stable or unstable & $8(26 \%)$ & $16(52 \%)$ & 0.02 & $13(65 \%)$ & 0.03 \\
\hline Recognizing signs of shock & $11(35 \%)$ & $19(61 \%)$ & 0.06 & $16(80 \%)$ & 0.07 \\
\hline Recognizing signs of sepsis & $3(10 \%)$ & $18(58 \%)$ & $<0.001$ & $10(50 \%)$ & 0.008 \\
\hline Recognizing and assessing altered mental status & $4(13 \%)$ & $18(58 \%)$ & $<0.001$ & $8(40 \%)$ & 0.02 \\
\hline $\begin{array}{l}\text { Assessment and management of patient with difficulty } \\
\text { breathing }\end{array}$ & $8(26 \%)$ & $22(71 \%)$ & $<0.001$ & $17(85 \%)$ & 0.002 \\
\hline Assessment and management of patient with fever & $14(45 \%)$ & $25(81 \%)$ & 0.005 & $18(90 \%)$ & 0.02 \\
\hline Suctioning the airway & $13(42 \%)$ & $21(68 \%)$ & 0.06 & $16(80 \%)$ & 0.008 \\
\hline Repositioning the airway & $8(26 \%)$ & $20(65 \%)$ & 0.004 & $14(70 \%)$ & 0.02 \\
\hline Inserting an oral airway & $6(19 \%)$ & $19(61 \%)$ & 0.001 & $13(65 \%)$ & 0.04 \\
\hline Inserting a nasopharyngeal airway & $6(19 \%)$ & $17(55 \%)$ & 0.007 & $11(55 \%)$ & 0.11 \\
\hline Managing a choking patient & $2(6 \%)$ & $17(55 \%)$ & $<0.001$ & $8(40 \%)$ & 0.07 \\
\hline Administering oxygen & $19(61 \%)$ & $22(71 \%)$ & 0.51 & $19(95 \%)$ & 0.03 \\
\hline Using a bag valve mask & $9(29 \%)$ & $20(65 \%)$ & 0.01 & $13(65 \%)$ & 0.02 \\
\hline Performing a needle thoracostomy & $2(6 \%)$ & $15(48 \%)$ & 0.001 & $1(5 \%)$ & 1.0 \\
\hline
\end{tabular}

* McNemar's exact chi-squared for paired data between baseline and immediate post-training 
Table 4 COVID-19 assessment at six months

\begin{tabular}{|c|c|}
\hline & $\begin{array}{l}\text { Participants } \\
(n=20)\end{array}$ \\
\hline Provided direct clinical care for patients with confirmed COVID-19 n (\%) & $8(40 \%)$ \\
\hline Provided direct clinical care for patients with suspected COVID-19 n (\%) & $18(90 \%)$ \\
\hline \multicolumn{2}{|l|}{ Participants reporting "strongly agree" } \\
\hline Have sufficient experience to manage patients with COVID-19 n (\%) & $9(45 \%)$ \\
\hline This course has improved ability to manage patients with COVID-19 n (\%) & $10(50 \%)$ \\
\hline \multicolumn{2}{|l|}{ Participants reporting "very comfortable" } \\
\hline Caring for patients with COVID-19 n (\%) & $10(50 \%)$ \\
\hline Performing an initial assessment of patients with confirmed or suspected COVID-19 n (\%) & $11(55 \%)$ \\
\hline Recognizing signs of severe COVID-19 n (\%) & $12(60 \%)$ \\
\hline Assessing respiratory status of patients with confirmed or suspected COVID-19 n (\%) & $11(55 \%)$ \\
\hline Administering oxygen to patients with confirmed or suspected COVID-19 n (\%) & $10(50 \%)$ \\
\hline Assessing patients with confirmed or suspected COVID-19 for signs of shock n (\%) & $8(40 \%)$ \\
\hline
\end{tabular}

and critical care skills immediately post-course and at six months, demonstrating the durability of the training's impact. Notably, improvements in immediate post-course knowledge were similar in magnitude to prior BEC assessments [10-12] despite broadening inclusion criteria to staff from general medical wards and intensive care units, suggesting a role for this course beyond the emergency unit. To our knowledge, this is only the second study to conduct long-term follow-up of BEC training [21].

This study was conducted early in the COVID-19 outbreak-the WHO declared a pandemic 11 days after completion of the trainings. Although only 8 (40\%) participants reported providing clinical care for patients with confirmed COVID-19, availability of COVID-19 diagnostics was very limited in Sierra Leone at the time of the six month follow-up. Therefore, the 18 (90\%) participants who reported treating patients with suspected COVID-19 are likely a more accurate estimate of the true number treating COVID-19. Half of respondents at month six "strongly agreed" that the course improved ability to manage patients with COVID-19, and confidence in tasks related to respiratory care improved, such as using a bag valve mask and overall confidence managing patients with difficulty in breathing and fever. However, the improvement in oxygen delivery confidence at six months may not be attributable to the BEC course and suggests that efforts to provide oxygen delivery training in Sierra Leone during the COVID-19 pandemic were successful outside of this study. Though not the initial intent of our study, our results suggest that the BEC course, designed for long-term emergency health systems strengthening, could be used to prepare frontline healthcare workers for COVID-19 care. Indeed, OpenWHO has since developed COVID-19 training modules drawing on BEC course content [22]. COVID-19 has reinforced the need to shift to a systems-focused approach that spans medical specialties. We have demonstrated a practical example of how to begin to implement that shift in human resources for health by using the BEC course to train both emergency unit and inpatient staff from multiple facilities, although this may also be achievable with other courses. More broadly, these results highlight the potential to strengthen health system resilience through emergency and critical care training for frontline healthcare workers. Compared with the five prior assessments of the BEC course [10-12, 20, 21], participants' baseline knowledge assessment scores were the among the lowest. However, the increase in knowledge assessment scores from baseline to immediate post-course was the highest recorded. The only study reporting knowledge assessment scores from longer term follow-up [21] reported an increase of $16 \%$ from baseline at seven months, which is similar to our observed increase of $19 \%$ at six months. Of note, we observed that most of the skills with reductions in confidence from immediate post-training to month six were in hands-on skills (log roll, fracture immobilization, needle thoracostomy). This suggests a potential future avenue of inquiry to better understand where refresher trainings would be most useful.

\section{Future directions}

These findings highlight the importance of training interventions to improve emergency and critical care capacity in LICs, for COVID-19 as well as long-term health systems strengthening. Future efforts should be directed at integration with national training curricula for all cadres of front-line healthcare workers to facilitate broader dissemination and maximize impact. Particular attention should be paid to training of the trainers to expedite 
implementation at scale. Future investigations should explore the impact of providing refresher trainings with an emphasis of hands-on skills.

\section{Limitations}

This study has several limitations. Sample size was limited to 31 participants from two locations in Sierra Leone and it is unclear whether the results would generalize to other LICs. Second, we assessed confidence in skills rather than measuring performance. Follow-up studies should examine patient management in the unit to evaluate if changes in care quality or outcomes occur. Third, there is potential for reporting bias if participants wanted to rate their confidence higher after the training course. However, participants' knowledge assessment scores also improved, which is encouraging. Fourth, we did not track other potential trainings that participants may have received between the BEC course and the six month follow-up. Additional trainings in oxygen delivery in response to the COVID-19 pandemic could explain why participants' confidence scores for administering oxygen were significantly higher at six months but not immediate post-training. It is unknown if other trainings could have affected six-month scores through recall bias. However, it is reassuring that the knowledge assessment results at six months are similar to previously published data [21].

Finally, the COVID-19 pandemic posed unique challenges to follow-up efforts. The original study design had called for in-person administration of instruments at six months. However, COVID-19 restrictions made this infeasible and the study protocol was revised to allow for the use of REDCap online survey tools. It is likely that using the online forms presented a barrier for some participants, either through mobile data costs or user interface. Indeed, there were five participants who completed the confidence instrument but did not proceed to the knowledge instrument. Although participants that were lost to follow-up at six months had lower post-course knowledge scores, the use of paired statistical tests allows reliable conclusions on the impact at six months for the 15 participants with follow-up data, if not for the entire group.

\section{Conclusions}

This study demonstrates that the BEC course can be used to train both emergency and inpatient healthcare workers with lasting impact. Additionally, the timing of the course early in the COVID-19 pandemic provided an opportunity to illustrate the strategic overlap between building human resource capacity for longterm health systems strengthening and a respiratory pandemic. Future efforts should focus on integrating the $\mathrm{BEC}$ course with the national training curriculum and on training of the trainers to allow broader dissemination and implementation at scale.
Abbreviations

ABCDE: Airway, breathing, circulation, disability, and exposure; AVPU: Alert, voice, pain, unresponsive; BEC: Basic emergency care; KGH: Koidu Government Hospital; LIC: Low-income country; MoHS: Ministry of Health and Sanitation; PIH: Partners In Health; WHO: World Health Organization.

\section{Supplementary Information}

The online version contains supplementary material available at https://doi. org/10.1186/s12913-022-07556-8.

Additional file 1. Supplement to: Applying the WHO-ICRC BEC course to train emergency and inpatient healthcare workers in Sierra Leone early in the COVID-19 outbreak

\section{Acknowledgements}

We thank the Sierra Leone Ministry of Health and Sanitation for their support and dedication to these important topics. Most of all, we wish to thank all study participants.

\section{Authors' contributions}

PDS was responsible for leading the overall conceptualization of the study, funding, design, implementation, data acquisition, database creation and management, data analysis, data interpretation, and drafting the first version of the manuscript. CK assisted study conceptualization, design, implementation, and editing all versions of the manuscript. DK assisted study conceptualization, design, implementation, data acquisition, and editing all versions of the manuscript. MB assisted study conceptualization, design, implementation, data acquisition, and editing all versions of the manuscript. NL assisted study design, implementation, data acquisition, database creation and management, and editing all versions of the manuscript. ML assisted study conceptualization, design, implementation, and editing all versions of the manuscript. MP assisted study conceptualization, design, implementation, and editing all versions of the manuscript. RBG assisted data interpretation, drafting the first version of the manuscript, and editing all versions of the manuscript. MJV assisted study design, implementation, and editing all versions of the manuscript. RHM assisted study conceptualization, funding, design, implementation, data interpretation, and editing all versions of the manuscript. JM assisted study conceptualization, funding, design, and editing all versions of the manuscript. SAR assisted study conceptualization, design, data interpretation, and editing all versions of the manuscript. NL and PDS had full access to the data. All authors read and approved the final manuscript.

\section{Funding}

PDS received funding support for this study from the Chest Foundation's Community Service Grant Honoring D. Robert McCaffree, MD, Master FCCP and Brigham and Women's Hospital Division of Pulmonary and Critical Care Medicine. The funders had no role in study design, data collection, data analysis, data interpretation, or writing of the report. The study also received internal support from Partners In Health-Sierra Leone (PIH-SL).

\section{Availability of data and materials}

The datasets used and/or analysed during the current study are available from the corresponding author on reasonable request.

\section{Declarations}

Ethics approval and consent to participate

All participants provided written informed consent. This study was approved by the Partners Institutional Review Board (Protocol \#: 2020P000209) and Sierra Leone's National Ethics and Scientific Review Committee.

\section{Consent for publication}

Not applicable

\section{Competing interests}

The authors declare that they have no competing interests. 


\section{Author details}

${ }^{1}$ Brigham and Womens Hospital, Division of Pulmonary and Critical Care Medicine, 75 Francis Street Massachusetts, Boston, MA 02115, USA. ${ }^{2}$ Harvard Medical School, Boston, MA, USA. ${ }^{3}$ Partners In Health, Boston, MA, USA. ${ }^{4}$ Partners In Health-Sierra Leone, Kono, Sierra Leone. ${ }^{5}$ Ministry of Health and Sanitation, Freetown, Sierra Leone. ${ }^{6}$ University of Chicago, Section of Emergency Medicine, Chicago, IL, USA. ${ }^{7}$ Brigham and Womens Hospital, Department of Emergency Medicine, Boston, MA, USA.

Received: 20 October 2021 Accepted: 27 January 2022

Published online: 14 February 2022

\section{References}

1. Cancedda C, Davis SM, Dierberg KL, Lascher J, Kelly JD, Barrie MB, et al. Strengthening health systems while responding to a health crisis: lessons learned by a Nongovernmental Organization during the Ebola virus disease epidemic in Sierra Leone. J Infect Dis. 2016;214(suppl 3):S153-S163. https://doi.org/10.1093/infdis/jiw345.

2. Shoman H, Karafillakis E, Rawaf S. The link between the West African Ebola outbreak and health systems in Guinea, Liberia and Sierra Leone: a systematic review. Glob Health. 2017;13(1):1.

3. Langer M, Portella G, Finazzi S, Chatenoud L, Lanini S, Vairo F, et al. Intensive care support and clinical outcomes of patients with Ebola virus disease (EVD) in West Africa. Intensive Care Med. 2018;44(8):1266-75.

4. Novel Coronavirus Pneumonia Emergency Response Epidemiology Team. The epidemiological characteristics of an outbreak of 2019 novel coronavirus diseases (COVID-19) in China. China CDC Wkly. 2020;2(8):113-22.

5. African COVID-19 Critical Care Outcomes Study (ACCCOS) Investigators. Patient care and clinical outcomes for patients with COVID-19 infection admitted to African high-care or intensive care units (ACCCOS): a multicentre, prospective, observational cohort study. Lancet. 2021;397(10288):1885-94. https://doi.org/10.1016/S0140-6736(21)004414. PMID: 34022988; PMCID: PMC8137309.

6. Gostin LO. Ebola: Towards an International Health Systems Fund. Lancet. 2013;384(9951):E49-51.

7. Jamison DT, Gelband H, Horton S, Jha P, Laxminarayan R, Mock CN, Nugent R, editors. Disease Control Priorities: Improving Health and Reducing Poverty. 3rd ed. Washington (DC): The International Bank for Reconstruction and Development / The World Bank; 2017 Nov 27. PMID: 30212058.

8. World Health Organization and the International Committee of the Red Cross. Basic emergency care: approach to the acutely ill and injured [Internet]. 2018 [cited 2021 Oct 2]. Available from: https://www.who.int/ publications/i/item/basic-emergency-care-approach-to-the-acutely-illand-injured

9. Hagos AA, Firew T, Gebreyesus A, Sambo BH, Reynolds TA. Ethiopia's quest to champion emergency care systems. Bull World Health Organ. 2019:97:582.

10. Broccoli MC, Dixon J, Skarpiak B, Phiri G, Muck AE, Calvello Hynes EJ. Application of the World Health Organization's Basic Emergency Care course in Zambia. Afr J Emerg Med. 2021;11(1):140-3.

11. Kivlehan SM, Dixon J, Kalanzi J, Sawe HR, Chien E, Robert J, et al. Strengthening emergency care knowledge and skills in Uganda and Tanzania with the WHO-ICRC Basic Emergency Care Course. Emerg Med J. 2021;38(8):636-42.

12. Olufadeji A, Usoro A, Akubueze CE, Aiwonodagbon BO, Strong J, Kivlehan SM, et al. Results from the implementation of the World Health Organization Basic Emergency Care Course in Lagos. Nigeria Afr J Emerg Med. 2021;11(2):231-6.

13. Statistics Sierra Leone. Sierra Leone 2015 Population and Housing Census: National Analytic Report [Internet]. Freetown; 2017 [cited 2021 Oct 2]. Available from: https://www.statistics.sl/images/StatisticsSL/Documents/ Census/2015/2015_census_national_analytical_report.pdf

14. UNDP. The 2020 Human Development Report [Internet]. New York, NY: United Nations Development Programme; 2020 [cited 2021 Oct 2]. Available from: http://hdr.undp.org/sites/default/files/hdr2020.pdf

15. Africa Health Organisation. Sierra Leone [Internet]. [cited 2021 Oct 2]. Available from: https://aho.org/countries/sierra-leone
16. Ministry of Health and Sanitation. Annual Health Sector Performance Report 2016 [Internet]. Freetown; 2016 [cited 2021 Oct 2]. Available from: https://www.afro.who.int/sites/default/files/2017-08/Sierra Leone Health Sector Performance Report 2016.pdf

17. Ministry of Health and Sanitation, Government of Sierra Leone. Human Resources For Health: Sierra Leone Country Profile [Internet]. Freetown; 2016 [cited 2021 Oct 2]. Available from: https://www.afro.who.int/sites/ default/files/2017-05/hrhprofile16.pdf

18. Coyle RM, Harrison HL. Emergency care capacity in Freetown, Sierra Leone: A service evaluation. BMC Emerg Med. 2015;15(1):1-9.

19. Harris PA, Taylor R, Thielke R, Payne J, Gonzalez N, Conde JG. Research electronic data capture (REDCap)-A metadata-driven methodology and workflow process for providing translational research informatics support. J Biomed Inform. 2009;42(2):377-81.

20. Tenner AG, Sawe HR, Amato S, Kalanzi J, Kafwamfwa M, Geduld H, et al. Results from a World Health Organization pilot of the Basic Emergency Care Course in Sub Saharan Africa. PLoS ONE. 2019;14(11):e0224257.

21. Straube S, Chang-Bullick J, Nicholaus P, Mfinanga J, Rose C, Nichols T, et al. Novel educational adjuncts for the World Health Organization Basic Emergency Care Course: A prospective cohort study. Afr J Emerg Med. 2020;10(1):30-4.

22. OpenWHO. Clinical management of patients with COVID-19: Initial approach to the acutely ill patient [Internet]. [cited 2021 Oct 2]. Available from: https://openwho.org/courses/clinical-management-COVID-19-initi al-approach

\section{Publisher's Note}

Springer Nature remains neutral with regard to jurisdictional claims in published maps and institutional affiliations.
Ready to submit your research? Choose BMC and benefit from:

- fast, convenient online submission

- thorough peer review by experienced researchers in your field

- rapid publication on acceptance

- support for research data, including large and complex data types

- gold Open Access which fosters wider collaboration and increased citations

- maximum visibility for your research: over $100 \mathrm{M}$ website views per year

At BMC, research is always in progress.

Learn more biomedcentral.com/submissions 